\title{
Development and Application of a Self-Referencing Gluc ose Microsensor for the Measurement of Glucose Consumption by Pancreatic $\beta$-Cells
}

\author{
Sung-Kwon J ung, ${ }^{\dagger}$ J ames R. Trimarchi, ${ }^{\ddagger}$ Richard H. Sanger, ${ }^{\dagger}$ and Peter J . S. Smith*,† \\ Marine Biological Laboratory, BioCurrents Research Center and Laboratory for Reproductive Medicine, \\ 7 MBL Street, Woods Hole, Massachusetts 02543
}

Glucose gradients generated by an artificial source and $\beta$-cells were measured using an enzyme-based glucose microsensor, 8- $\mu \mathrm{m}$ tip diameter, as a self-referencing electrode. The technique is based on a difference measurement between two locations in a gradient and thus allows us to obtain real-time flux values with minimal impact of sensor drift or noise. Flux values were derived by incorporation of the measured differential current into Fick's first equation. In an artificial glucose gradient, a flux detection limit of $8.2 \pm 0.4 \mathrm{pmol} \cdot \mathrm{cm}^{-2} \cdot \mathrm{s}^{-1}$ (mean \pm

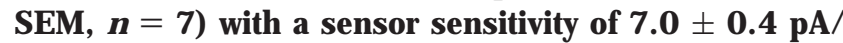
$\mathrm{mM}$ (mean $\pm \mathrm{SEM}, \mathbf{n}=16$ ) was demonstrated. Under biological conditions, the glucose sensor showed no oxygen dependence with $5 \mathrm{mM}$ glucose in the bulk medium. The addition of catalase to the bulk medium was shown to ameliorate surface-dependent flux distortion close to specimens, suggesting an underlying local accumulation of hydrogen peroxide. Glucose flux from $\beta$-cell clusters, measured in the presence of $5 \mathrm{mM}$ glucose, was $61.7 \pm 9.5 \mathrm{fmol} \cdot \mathrm{nL}^{-1} \cdot \mathrm{s}^{-1}$ (mean $\pm \mathrm{SEM}, \mathrm{n}=9$ ) and could be pharmacologically modulated. Glucose consumption in response to FCCP $(1 \mu \mathrm{M})$ transiently increased, subsequently decreasing to below basal by $93 \pm 16$ and 56 $\pm 6 \%$, respectively (mean $\pm S E M, n=5$ ). Consumption was decreased after the application of $10 \mu \mathrm{M}$ rotenone by $74 \pm 5 \%$ (mean $\pm S E M, n=4)$. These results demonstrate that an enzyme-based amperometric microsensor can be applied in the self-referencing mode. Further, in obtaining glucose flux measurements from small clusters of cells, these are the first recordings of the real-time dynamic of glucose movements in a biological microenvironment.

Glucose is important to many cells as it serves in metabolic energy production, but despite a strong interest in glucose transport and consumption, ${ }^{1}$ real-time measurement of glucose flux has not been reported. To date, glucose consumption has been measured using, for example, the radiochemistry of ${ }^{3} \mathrm{H}_{2} \mathrm{O}$

* Corresponding author: (e-mail) psmith@mbl.edu; (voice) 508-289-7241/ 7265 (fax) 508-540-6902.

† BioCurrents Research Center.

₹ Laboratory for Reproductive M edicine.

(1) Carruthers, A.; Zottola, R. J. Transport Processes in Eukaryotic and Prokaryotic Organisms; Elsevier: New York, 1996; Chapter 14, Vol. 2. production from $\mathrm{D}_{-}\left[5_{-3}^{-3} \mathrm{H}\right]$ glucose metabolism, ${ }^{2}{ }^{14} \mathrm{CO}_{2}$ production from $\left[{ }^{14} \mathrm{C}\right]$ glucose oxidation, ${ }^{3}$ or microfluorometry of glucose and 2-deoxyglucose uptake using a cocktail of hexokinase, ATP, glucose 6-phosphate, and NADP. ${ }^{4}$ However, glucose can be converted to produce the easily detectable compound hydrogen peroxide by utilizing the enzyme glucose oxidase (GOx). The process requires oxygen as a cosubstrate and is as follows:

$$
\text { glucose }+\mathrm{O}_{2} \stackrel{\mathrm{GOx}}{\longrightarrow} \text { gluconolactone }+\mathrm{H}_{2} \mathrm{O}_{2}
$$

The production of $\mathrm{H}_{2} \mathrm{O}_{2}$ is measured at a charged platinum electrode surface: ${ }^{5}$

$$
\mathrm{H}_{2} \mathrm{O}_{2} \stackrel{+600 \mathrm{mV} \text { vs Ag/ AgCl}}{\longrightarrow} \mathrm{O}_{2}+2 \mathrm{H}^{+}+2 \mathrm{e}^{-}
$$

The use of glucose oxidase-based electrodes is a wellestablished method for the detection of in vivo levels of circulating glucose $e^{6,7}$ although in these applications the electrodes are usually greater than $200 \mu \mathrm{m}$ in tip diameter. In the study presented here, the same reaction is used but the electrode has been miniaturized to $\sim 8 \mu \mathrm{m}$ in tip diameter and applied in a self-referencing mode, a technique previously described for the detection of ion and gas flux frequently at the single-cell level. $8,9,10$ The self-referencing technique extracts analytically useful data from raw signals by measuring the difference values between two known positions because the drift or noise on the electrode is relatively common to both positions (see Figure 1). This approach belongs to a family of techniques employing position modulation of microprobes to enhance detection. Variants can be seen in scanning electrochemical microscopy (SECM), ${ }^{11}$ atomic force microscopy (AFM), 12 and

(2) Guillam, M.-T.; Dupraz, P.; Thorens, B. Diabetes 2000, 49, 1485-1491.

(3) M CD aniel, M. L.; King, S.; Anderson, S.; Fink, J.; Lacy, P. E. Diabetologia 1974, 10, 303-308.

(4) Wree, A. Eur. J. Morphol. 1990, 28, 132-138.

(5) Guilbault, G. G.; Lubrano, G. J. Anal. Chim. Acta 1973, 64, 439-455.

(6) Zhang, Y.; Wilson, G. S. Anal. Chim. Acta 1993, 281, 513-520.

(7) Hu, Y.; Wilson, G. S. J. Neurochem. 1997, 68, 1745-1752.

(8) Kumar, S. M.; Porterfield, D. M.; M uller, K. J.; Smith, P. J. S.; Sahley, C. L. J. Neurosci. 2001, 21, 215-220.

(9) Land, S. C.; Porterfield, D. M.; Sanger, R. H.; Smith, P. J. S. J. Exp. Biol. 1999, 202, 211-218.

(10) Smith, P. J. S.; Trimarchi, J. R. Am. J. Physiol. 2001, 280, C1-C11.

(11) Wipf, D. O.; Bard, A. J. Anal. Chem. 1992, 64, 1362-1367.

(12) Siedlecki, C. A.; M archant, R. E. Biomaterials 1998, 19, 441-454. 
A

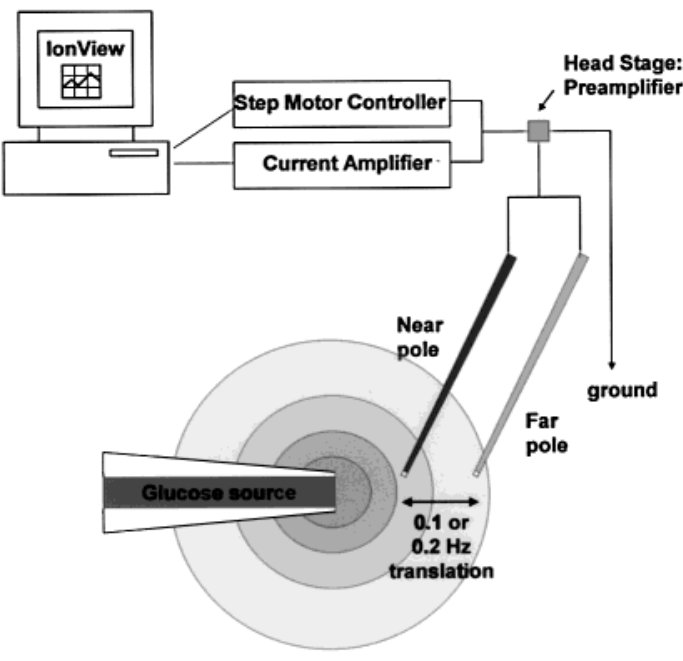

B

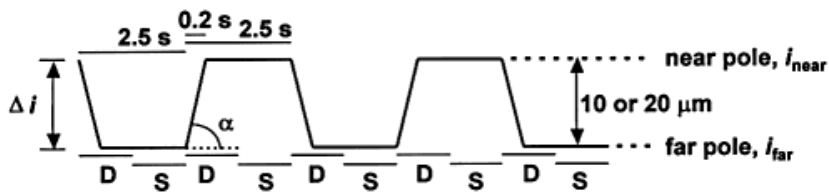

Figure 1. (A) Operation of the self-referencing glucose microsensor. A personal computer controls the translational square wave frequency of sensor positioning allowing lonView software to report current changes coherent with sensor movement in a gradient. The positioning of the sensor and its motion relative to the specimen is guided by observation on a video monitor. (B) Time-dependent sensor location and data processing in lonView software. In this example, the frequency of translation was set at $0.2 \mathrm{~Hz}$. IonView automatically subtracts the current measured at the far pole or position from that measured at the near pole or position. While the first half of the values (denoted as D) at each position is discarded to minimize any artifacts from sensor motion and gradient disturbance, the second half (denoted as $\mathrm{S}$ ) is sampled for mathematical subtraction. The speed of sensor movement is set by $\alpha$.

scanning reference electrode techniques used in both biological and materials sciences. ${ }^{13,14}$

In the present study, glucose flux measurements are made from small aggregates of a cultured cell line comprising $\beta$-pancreatic cells ( $\beta$-cell) derived from the hamster (hamster insulinoma tumor, HIT). Glucose transport is a common and important component of cell metabolism, but the $\beta$-cell is particularly interesting as it plays a role in insulin secretion and the clinical condition of type II diabetes. In these cells, the movement of glucose from the blood across the plasma membrane is a key component in the regulation of insulin secretion, the chemical regulator of blood glucose concentration. ${ }^{15}$ To date there has been no method available for the real-time measurement of glucose uptake from the microenvironment surrounding these and other cells.

(13) Nuccitelli, R. Vibrating Probe Technique for Studies of Ion Transport. In Noninvasive Techniques in Cell Biology; Foskett, J. K., Grinstein, S., Eds.; Wiley-Liss, Inc.: New York, 1990; pp 273-310.

(14) Kinlen, P. J.; M enon, V.; Ding, W. J. Electrochem. Soc. 1999, 146, 36903695.

(15) Schuit, F. C.; Huypens, P.; Heimberg, H.; Pipeleers, D. G. Diabetes 2001 $50,1-11$.
A

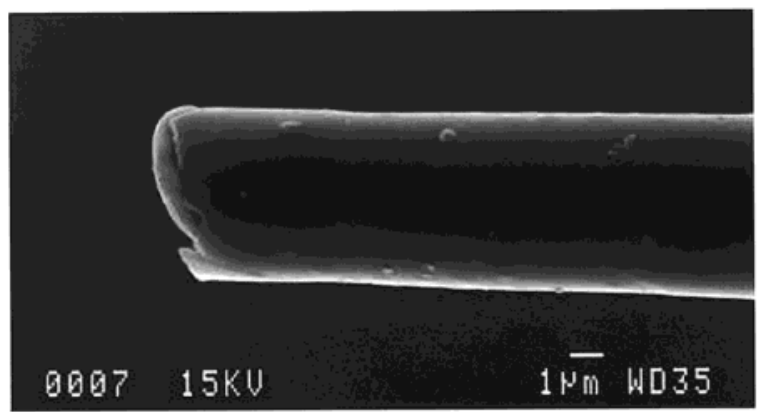

B

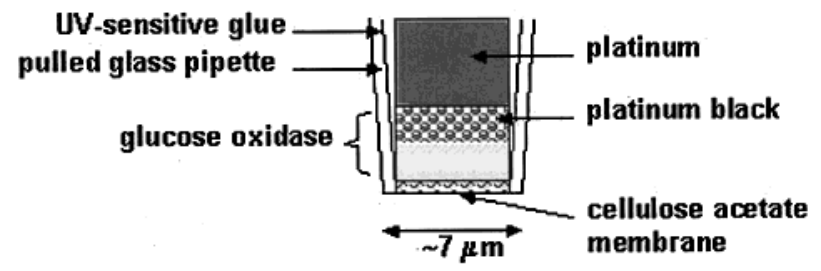

Figure 2. (A) Scanning electron micrograph of the glucose microsensor tip. (B) Illustration of glucose microsensor construction.

The results of this study show that enzyme-based sensors can be incorporated into a self-referencing mode delivering high temporal and spatial resolution along with a threshold of flux detection above background of $8.2 \mathrm{pmol} \cdot \mathrm{cm}^{-2} \cdot \mathrm{s}^{-1}$ against a bulk glucose concentration of $1 \mathrm{mM}$. Biological glucose flux data are shown to be responsive to pharmacological manipulation.

\section{EXPERIMENTAL SECTION}

Fabrication of Glucose and Oxygen Ultramicrosensors. Glucose and oxygen microsensors were prepared as described previously with slight modification (see Figure 2). ${ }^{16,17}$ Glucose microsensors were fabricated by gluing a $25-\mu \mathrm{m}$-diameter platinum wire (1.5-cm length; Johnson M atthey, Ward Hill, MA) into 25gauge hypodermic tubing ( $5 \mathrm{~cm}$ long; Small Parts, Inc., M iami Lakes, FL) using silver epoxy from Epoxy Technology, Inc. (Billerica, M A). The wire was electrochemically etched down to $\sim 4-\mu \mathrm{m}$ diameter in an aqueous solution of $4 \mathrm{M} \mathrm{KCN}$ and $1 \mathrm{M}$ $\mathrm{NaOH}$ by applying 4.0- $\mathrm{V}$ square waves for $8 \mathrm{~min}$ at a frequency of $250 \mathrm{~Hz}$ using a function generator (Leader Instruments, Hauppauge, NY). The etched Pt wires were inserted into pulled glass capillaries (1.5-mm 0.d.; TW150-4; World Precision Instruments, Sarasota, FL) and filled with UV-curable optical adhesive (Norland Products Inc., New Brunswick, NJ) so that the etched wires protruded from the pipet tip by $\sim 10 \mu \mathrm{m}$. The adhesive was cured under UV light for $20 \mathrm{~min}$. The exposed Pt wires were etched again to form a cavity $\sim 5 \mu \mathrm{m}$ deep. Once etched, Pt particles were electrochemically plated on the recessed Pt wire at $-0.2 \mathrm{~V}$ versus $\mathrm{Ag} / \mathrm{AgCl}$ in an aqueous solution of $10 \mathrm{mM}$ hydrogen hexachloroplatinate(IV) hydrate (20,608-3; Aldrich, M ilwaukee, WI) for 3-5 min. The amount of charge required for

(16) Jung, S.-K.; Gorski, W.; Aspinwall, C. A.; Kauri, L. M .; Kennedy, R. T. Anal. Chem. 1999, 71, 3642-3649.

(17) Jung, S.-K.; Kauri, L. M.; Qian, W.-J.; Kennedy, R. T. J. Biol. Chem. 2000, $275,6642-6650$

3760 Analytical Chemistry, Vol. 73, No. 15, August 1, 2001 
platinization was $(1.2 \pm 0.3) \times 10^{-5} \mathrm{C}($ mean $\pm \mathrm{SD}, \mathrm{n}=26)$. Glucose oxidase (GOx; EC 1.1.3.4., type X-S from Aspergillus niger, Sigma, St. Louis, M O) was then loaded onto the platinum particles by immersing the tip of the electrodes in $1 \%(w / v)$ aqueous $\mathrm{GOx}$ solution for $10 \mathrm{~min}$. The enzyme was immobilized with the vapor of $10 \%$ glutaraldehyde for $1 \mathrm{~min}$. The immobilized enzyme layer was coated with $10 \%$ (w/ v in acetone) $30-k D$ a cellulose acetate by dipping ( $10 \mathrm{~s}$ ) and drying ( $60 \mathrm{~s})$, repeated 4 times. All solutions were made up in distilled water (Milli-Q Plus; Millipore Co, Bedford, M A). The final tip diameter was $\sim 8 \mu \mathrm{m}$.

Oxygen sensors were fabricated with platinum wires etched down to $\sim 1-\mu \mathrm{m}$ diameter, inserted into pulled glass capillaries (tip diameters $3 \mu \mathrm{m}$ ) filled with optical adhesive, cured as above, and recessed by electroetching to form a cavity a $\sim 2-\mu \mathrm{m}$-deep cavity. The oxygen electrodes were then coated with $10 \%$ cellulose acetate. The resulting sensor geometry resembles that of the glucose sensor except in size. The final tip diameter was $\sim 3 \mu \mathrm{m}$.

Operation and Testing of Oxygen and Glucose Sensors. Calibration and measurement of the response times of the sensors were performed in a solution consisting of $20 \mathrm{mM}$ HEPES buffer with $0.15 \mathrm{M} \mathrm{NaCl}$ at $\mathrm{pH} 7.4$ by employing micropuffing ( $\sim \mu \mathrm{L}$ ) s) or a perfusion system $(\sim 32 \mu \mathrm{L} / \mathrm{s})$. For all measurements, glucose and oxygen sensors were polarized at +0.60 and -0.60 $\mathrm{V}$ versus $\mathrm{Ag} / \mathrm{AgCl}$ reference electrode, respectively, and conditioned for at least $1 \mathrm{~h}$ in $5 \mathrm{mM}$ glucose solutions. Sensors drifting in excess of $\pm 5 \% \mathrm{~h}$ were not employed for measurements. Glucose sensitivity was tested using standard solutions at different glucose concentrations. The influence of oxygen on glucose microsensor performance was tested by measuring both glucose and oxygen with their respective sensors, while changing oxygen content in the perfused solution. Normal air-saturated solutions containing $210 \mu \mathrm{M}$ oxygen could be deoxygenated by bubbling with argon gas.

Self-Referencing Glucose Microsensor System. The experimental platform was constructed around an Axiovert 10 (Carl Zeiss Inc.) inverted microscope fitted with a stage plate upon which the translational motion control system, carrying the headstage amplifier and sensor, was mounted. ${ }^{18}$ The motion control system consisted of Newport 310 series translation stages arranged in an orthogonal array and driven by size 23 linear stepper motors whose movement were controlled by the IonView software (BioCurrents Research Center (BRC) MBL, Woods Hole MA). This arrangement provided nanometer resolution of movement ${ }^{19}$ of the head stage along with the glucose sensor in either a square wave oscillation or single-step mode. The sensor positioning relative to a biological target was observed with bright-field optics and guided remotely by viewing with a video camera (Burle TC655EAC). The entire assembly was mounted on an antivibration table (Technical M anufacturing Corp., Peabody, M A) and housed within a thermally insulated Faraday box, maintained at $37^{\circ} \mathrm{C}$ with a temperature control system. Data were collected using IonView (BRC, M BL, Woods Hole) at $1000 \mathrm{~Hz}$ or Axoscope (Axon Instruments, Foster City, CA) at $300 \mathrm{~Hz}$ with a low-pass cutoff frequency of $10 \mathrm{~Hz}$. The motion controllers, temperature controller, and amplifiers, as well as coordinating software, are products

(18) Smith, P. J. S.; Hammar, K.; Porterfield, D. M .; Sanger, R. H.; Trimarchi, J. R. Microsc. Res. Technol. 1999, 46, 398-417.

(19) Danuser, G. Bull. Swiss Soc. Micro-Tech., Lausanne 1998, 22, 34-39. of the BioCurrents Research Center ( $M$ arine Biological Laboratory, Woods Hole, M A., www.mbl.edu/ BioCurrents).

Glucose Flux Measurement. To study the utility of the selfreferencing glucose sensor for flux measurement, a glucose gradient was artificially generated from a glass micropipet (tip diameter $\sim 10 \mu \mathrm{m}$ ) holding a buffered solution of 500 or $99 \mathrm{mM}$ glucose and $1 \mathrm{mM}$ mannose in a $0.5 \mathrm{wt} \% \mathrm{v}$ agar solution. Agar was found to be useful for preventing capillary movement of solution between the pipet and bath. To further avoid this the source, the pipet was held in a closed-volume micropipet holder (World Precision Instruments). To counterbalance the osmotic strength of the source pipet, a buffer solution with $500 \mathrm{mM}$ mannose or $99 \mathrm{mM}$ mannose with $1 \mathrm{mM}$ glucose was used in the bath solution (volume of $4 \mathrm{~mL}$ ). M annose, an epimer of glucose, was not detected by the glucose sensor (not shown). A gradient of glucose around the source pipet was allowed to stabilize for 30 min. Measurements within this gradient were made at several points by electrodes in stationary and self-referencing modes. The sensor was referenced to ground via a return $3 \mathrm{M} \mathrm{KCl}$ and $3 \%$ agar bridge placed in the bulk medium. Sensor output prior to calculation of a differential (see below) includes the background signal caused by the analyte concentration in the bulk as well as fluctuations within the system, particularly between the sensor and the return electrode. Total current, therefore, represents the sum of all sources, bulk glucose concentration, drift, and biological flux.

In the self-referencing mode, the glucose microsensor was laterally oscillated between two points 10 or $20 \mu \mathrm{m}$ apart at a square wave frequency of $0.1,0.15,0.2$, or $0.3 \mathrm{~Hz}$. Five-tenths of the values measured from each sensor position were discarded to eliminate movement artifacts, errors arising from response time limitations, and the period of gradient reestablishment after translation (see Results and Discussion). The IonView software reports both total current $(\mathrm{i})$ and differential current $(\Delta \mathrm{i})$. Total current (i) is used to calibrate the sensor. By convention $\Delta \mathrm{i}$ is defined as $\left(i_{\text {near }}-i_{\text {far }}\right)$, where $i_{\text {near }}$ is the current at the near position/ pole from a target and $i_{\text {far }}$ is the value at the farthest position, far pole, from the same target.18 The term self-referencing arises from the procedure of comparing the signal between sensor and ground at each pole of translation and calculating the differential $(\Delta \mathrm{i})$, thereby removing the comparatively large background signal and drift, common to both poles of translation. This procedure allows the selective amplification of the differential signal. A summary of the system setup and data processing is illustrated in Figure 1.

Flux measurements around HIT cell clusters were done in HEPES-buffered solution containing $118 \mathrm{mM} \mathrm{NaCl}, 5.4 \mathrm{mM} \mathrm{KCl}$, $2.4 \mathrm{mM} \mathrm{CaCl} 2,1.2 \mathrm{mM} \mathrm{MgSO}_{4}, 1.2 \mathrm{mM} \mathrm{KH}_{2} \mathrm{PO}_{4}$, and $5 \mathrm{mM}$ $\mathrm{NaHCO}_{3}$. The outer surface of the cluster served as a reference point for sensor positioning, and distance measurements were made relative to this point. The glucose microsensor was positioned adjacent to the cluster and self-referenced perpendicularly to the contour of the expected glucose gradient. To ensure that the sensor performance was independent from oxygen, oxygen levels were reversed by placing an oxygen source ( $100 \%$ oxygen) $10 \mu \mathrm{m}$ from the cell cluster and $\sim 50 \mu \mathrm{m}$ from the glucose sensor. In situ calibration of the sensor was performed immediately after the flux measurements. The data were only 
accepted if the sensor drift showed less than $\pm 5 \%$ over the experimental period. Typically, this was $30 \mathrm{~min}$. Therefore, any change in electrode sensitivity during the experiment should have a minimal impact on the final quantification of the data. All data were reported as means \pm SEM unless otherwise mentioned.

Oxygen Measurements. The measurement and analysis described above for the glucose sensor output was first developed for an amperometric sensors without the enzyme. Land et al. first reported on the use of a self-referencing oxygen electrode for monitoring oxygen consumption from a single cultured neuron. ${ }^{9}$ In that study, commercially available oxygen electrodes were used (Diamond General Development Corp., Ann Arbor, MI). In the present study, the oxygen electrodes were built as described above.

Cell Culture. Pancreatic HIT cells, donated from the laboratory of Dr. B. Corkey, Boston M edical Center, Boston University, were incubated in a culture medium composed of RPMI 1640 solution, $10 \mathrm{mM}$ HEPES, 10\%fetal bovine serum, $2 \mathrm{mM}$ glutamine, and $1 \%$ penicillin-streptomycin at $37{ }^{\circ} \mathrm{C}$ in humidified $95 \%$ air and $5 \% \mathrm{CO}_{2}$ and handled as described previously. ${ }^{20} \mathrm{~F}$ or trypsinization and splitting, trypsin-EDTA solution was added to a culture flask and incubated for $30 \mathrm{~s}$ while triturating with a transfer pipet. Serum was added to stop the digestive action of trypsin. Supernatant was removed after centrifugation at $1000 \mathrm{rpm}$ for $2 \mathrm{~min}$. Culture medium was added, and cells were plated into glass-bottom Petri dishes for incubation and subsequent observation. Petri dishes (35-mm diameter) were from Matek (Ashland, MA). All cell culture media and solutions were from Gibco (Grand Island, NY). All other chemicals were purchased from Aldrich or Sigma Chemical Co.

Cell clusters, approximating to spheroids, appear approximately two weeks after splitting. Their sizes ranged from 70 to $300 \mu \mathrm{m}$ in diameter depending on the time in culture.

\section{RESULTS AND DISCUSSION}

Characterization of the Glucose Microsensor and Validation of Its Application to the Self-Referencing Technique. A differential current $(\Delta i)$ is acquired by self-referencing the glucose microsensor in the glucose diffusion gradient surrounding either an artificial source or respiring tissue as described above. The value $\Delta \mathrm{i}$ can be converted into a concentration difference $(\Delta C)$ by reference to the electrode calibration (see below). The resulting concentration difference can be converted into a flux measurement according to Fick's first law:

$$
\mathrm{J}=-\mathrm{D}_{\mathrm{G}}(\Delta \mathrm{C} / \Delta \mathrm{r})
$$

where $J$ is flux (in $\mathrm{mol} \cdot \mathrm{cm}^{-2} \cdot \mathrm{s}^{-1}$ ), $D_{G}$ is the diffusion coefficient of glucose $\left(6.7 \times 10^{-6} \mathrm{~cm}^{2} \cdot \mathrm{s}^{-1}\right),{ }^{21}$ and $(\Delta \mathrm{C} / \Delta \mathrm{r})$ is the concentration difference over the distance of electrode translation $(\Delta r)$. Since the mechanism of glucose sensing, based on glucose oxidase, involves oxygen (see eqs 1 and 2) and oxygen consumption is frequently accompanied by glucose consumption in living cells, it is of importance to determine the oxygen dependency of the

(20) Santerre, R. F.; Cook, R. A.; Crisel, R. M.; Sharp, J. D.; Schmidt, R. J.; Williams, D. C.; Wilson, C. P. Proc. Natl. Acad. Sci. U.S.A. 1981, 78, 43394343.

(21) Weast, R. C., Ed. Handbook of Chemistry and Physics; CRC Press: Boca Raton, $\mathrm{FL}, 1975$.
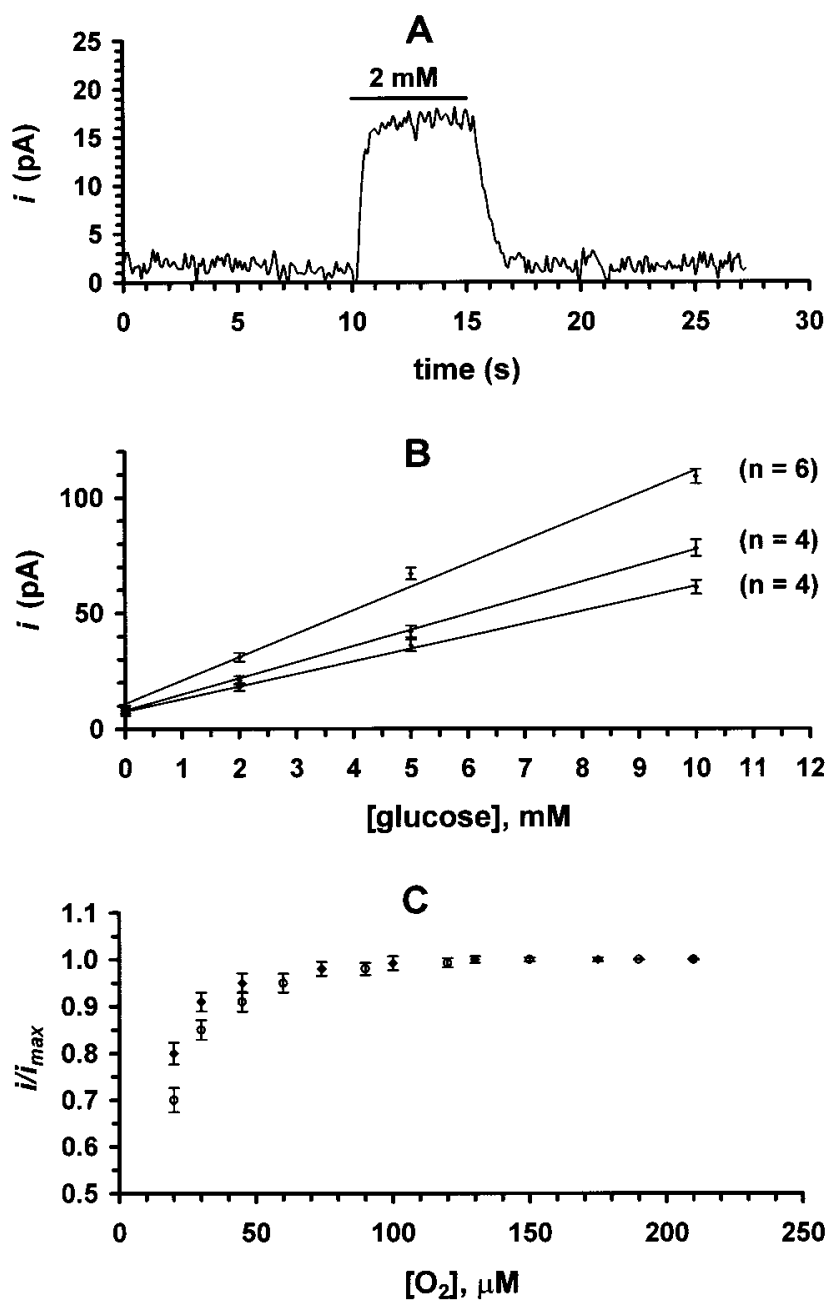

Figure 3. Glucose microsensor characterization. (A) Current recorded during puffing a $2 \mathrm{mM}$ glucose solution, with oxygen levels equilibrated to air, onto the sensor for $5 \mathrm{~s}$. (B) Calibration of three different glucose microsensors. All of the measurements were performed in air-saturated buffer. For a given sensor, the measurements at each concentration were repeated the number of times indicated in parentheses. (C) Oxygen influence on current response $(n=5)$. The glucose signal was taken as the current at a given oxygen level $(I)$ relative to that obtained at $210 \mu \mathrm{M}\left(i_{\max }\right) . \diamond$, at $2 \mathrm{mM}$ glucose; $\mathrm{O}$, at $5 \mathrm{mM}$ glucose. For all experiments, the applied potential was $+0.6 \mathrm{~V}$ vs $\mathrm{Ag} / \mathrm{AgCl}$ and the background solution was $50 \mathrm{mM} \mathrm{HEPES}$ with $0.15 \mathrm{M} \mathrm{NaCl}$ at pH 7.4 at $37^{\circ} \mathrm{C}$. Error bars represent 1 standard deviation.

self-referencing glucose system. In addition, a glucose sensor needs to respond reasonably rapidly to new glucose concentrations when operated in a self-referencing mode, as the sensor resides at each measurement position for just over $1 \mathrm{~s}$. Considering these requirements, we adopted a previously published glucose electrode design (Figure 2$)^{17}$ which shows acceptable oxygen dependence as well as a fast response time.

The sensor response time ( $t_{90}$, time for signal change from 10 to $90 \%$ of maximum), measured in a steep gradient without translation or signal processing, was $0.46 \pm 0.07 \mathrm{~s}$ ( $n=6$; Figure $3 A)$. These data show that the glucose sensor itself responded quickly to the changes in glucose level. Hu and Wilson reported a response time of $5.2 \mathrm{~s}$ with an implantable glucose sensor (cylindrical reactive surface of $110-\mu \mathrm{m}$ diameter and $500-\mu \mathrm{m}$ length) with thick inner and outer membranes. ${ }^{7}$ The smaller 


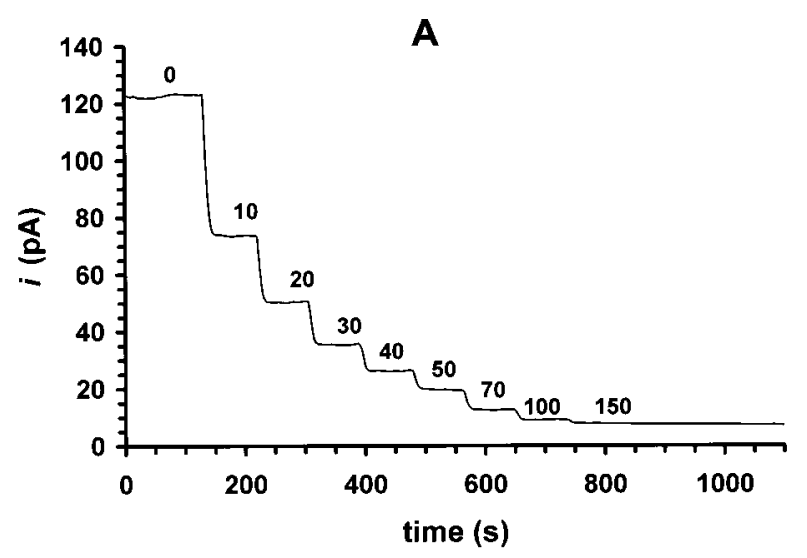

B

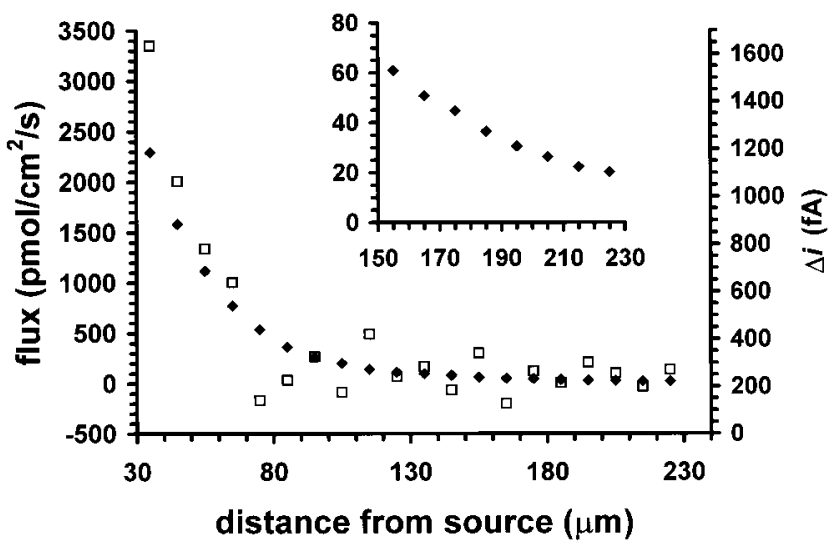

Figure 4. (A) Real-time trace of glucose gradient generated from a $500 \mathrm{mM}$ glucose source. The measurement was made by using a stationary glucose microsensor. The numbers indicate the sensor locations from a source pipet (diameter $\sim 10 \mu \mathrm{m}$ ). (B) Illustration of the effectiveness of the self-referencing technique. Glucose flux values were measured from $99 \mathrm{mM}$ glucose source (diameter $\sim 10$ $\mu \mathrm{m})$ using a self-referencing sensor with a $10-\mu \mathrm{m}$ excursion at a frequency of $0.2 \mathrm{~Hz}(\bullet)$ or with the sensor held in stationary mode at both poles of translation ( $\square$ ). From these two stationary values, a current difference can be calculated and flux determined. The sensor was repositioned in a sequence of $10-\mu \mathrm{m}$ steps moving away from the source. Note that values derived from stationary measurements can only follow the gradient within $70 \mu \mathrm{m}$ of the source. After that point the values appear random. In contrast, the self-referencing data continue to follow the gradient even at distances in excess of 200 $\mu \mathrm{m}$ (inset). The mismatch between differential measurements extracted from stationary values versus those measured by selfreferencing is discussed in the text.

design reported in the present study has, clearly, a decreased time to t9o. In calibration against known solutions of glucose, the sensor response was linear up to $10 \mathrm{mM}$ glucose while slight deviations were observed with sensors exhibiting a high sensitivity (see Figure $3 \mathrm{~B}$ ). The average sensitivity of the sensors was $7.0 \pm 0.4$ $\mathrm{pA} / \mathrm{mM}(\mathrm{n}=16)$. For $5 \mathrm{mM}$ glucose, the sensor signal was independent of the oxygen concentration as long as the oxygen concentration was above $148 \pm 5 \mu \mathrm{M}(\mathrm{n}=9$; Figure $3 \mathrm{C})$. For 2 $\mathrm{mM}$ glucose, the interference by an oxygen limitation was less and freedom from an oxygen influence was observed at oxygen concentrations above $97 \pm 4 \mu \mathrm{M}(\mathrm{n}=4)$.

To validate the application of the glucose sensor to flux measurements, a glucose gradient was artificially generated as described above. A glucose sensor was then positioned without
Table 1. Influence of the Self-Referencing Frequency on the Compensation Factor $\left(C_{\mathrm{f}}\right)^{a}$

$\begin{gathered}\text { frequency of the } \\ \text { self-referencing }(\mathrm{Hz})\end{gathered}$
0.10
0.15
0.20
0.30

$$
\begin{gathered}
\begin{array}{c}
\text { compensation } \\
\text { factor }\left(C_{f}\right)^{b}
\end{array} \\
1.20 \pm 0.04(n=5) \\
1.32 \pm 0.03(n=5) \\
1.46 \pm 0.09(n=6) \\
1.51 \pm 0.16(n=5)
\end{gathered}
$$

${ }^{a}$ The $C_{f}$ was obtained by comparing the difference values between two positions measured in the stationary mode with those measured in the self-referencing mode. ${ }^{b}$ The values are expressed as mean \pm SEM with the number of sensors tested $(n)$.

translation (stationary mode) within the gradient and the total current recorded at several positions (Figure 4A). The traces in Figure $4 \mathrm{~A}$ represent the glucose levels at given distances from the glucose source pipet and demonstrate that the glucose sensor is suitable for gradient measurements. To test the effectiveness of the self-referencing technique, the glucose fluxes were measured in the self-referencing and stationary mode (Figure 4B). As shown in Figure $4 \mathrm{~B}$, the flux values measured in the selfreferencing mode before compensation, particularly those collected near the source $(\bullet)$, are smaller than those calculated from data collected in a stationary mode ( $\square$ ). This disparity is a frequency-dependent underestimate of $\Delta \mathrm{i}$ between those values expected by comparison of total current in a steep gradient and those measured during translation. As the limitation should not be in the response time of the sensor or electronics, it seems more likely to result from limitations on reestablishing the full gradient after movement, which must inevitably produce local mixing. As has been demonstrated with other self-referencing sensors, ${ }^{18}$ this disparity can be compensated for (Table 1 and below). Therefore, to calculate the flux, the $\Delta \mathrm{C}$ in eq 3 is replaced by $\left(C_{f} \Delta \mathrm{i}\right) / \mathrm{S}$, which is obtained in the self-referencing mode as follows:

$$
J=-D_{G}\left(C_{f} \Delta i / S \Delta r\right)
$$

where $C_{f}$ is a compensation factor and $S$ is the sensitivity of the glucose sensor (in $\mathrm{pA} / \mathrm{mM}$ ), while other notations are the same as eq 3. $C_{f}$ varies such that the slower the frequency, the smaller the $C_{f}$ (see Table 1)-the glucose signal will more closely approximate the full response as the sensor stays longer in a position. However, limitations caused by the frequency of translation must be balanced against remaining at one measurement position too long and increasing the impact of drift, which becomes more of a problem with shallower gradients. As currently designed, the system is optimized to operate at a range of $0.2-$ $0.3 \mathrm{~Hz}$. A square wave translational frequency of $0.2 \mathrm{~Hz}$ is used for the flux measurements in pancreatic HIT cells. In stationary mode ( $\square$ ), flux values smaller than $1 \mathrm{nmol} \cdot \mathrm{cm}^{-2} \cdot \mathrm{s}^{-1}$ are hard to measure because of the sensor drift and noise (Figure 4B). On the other hand, in the self-referencing mode, fluxes as low as 8.2 $\pm 0.4 \mathrm{pmol} \cdot \mathrm{cm}^{-2} \cdot \mathrm{s}^{-1}(\mathrm{n}=7)$ can be reliably measured (Figure $4 B$ and inset). This flux value was derived from $\Delta \mathrm{i}$ of $9.8 \mathrm{fA}$. Signals of this magnitude could not be obtained from stationary electrochemical sensors. Signal averaging can be used in the analysis of $\Delta \mathrm{i}$ to further improve the signal quality by reducing 
the impact of noise and transient drift, independent of changes in sensitivity, which are minimized by the self-referencing technique. However, a greater problem will arise from changes in electrode sensitivity over the recording period. The flux calculation in eq 4 is valid under the condition that the sensor sensitivity (S) remains constant during measurement. Although by constantly monitoring both the total (i) and difference current $(\Delta \mathrm{i})$, as done by the IonView software, it is possible to incorporate time-dependent sensitivity changes, in the current study we simply used a rejection criterion (see Experimental Section). In fact, for the experimental data presented here, the glucose sensors drifted by only $2.8 \pm$ $1.8 \%$ in 30 min (mean $\pm S D, n=16$ ).

Application of the Self-R eferencing Glucose Sensor to the Flux Measurement around a B iological Specimen: Examination of B oundary Artifacts. In a diffusion-determined environment, the depletion of glucose generated by cellular consumption will be highest around the cell surface and gradually decrease with increasing distance from the cell. As glucose is being consumed, $\Delta \mathrm{i}$ should be negative as $i_{\text {near }}$ will be less than $i_{\text {far }}$. However, in practice, a positive value for $\Delta \mathrm{i}$ was recording in close proximity to the cells, implying an improbable production of glucose. This phenomenon can be explained by considering the chemistry and limitations of the glucose sensor. In the presence of oxygen, glucose transported through the cellulose acetate membrane is oxidized by glucose oxidase and converted to gluconolactone and hydrogen peroxide (Figure $5 \mathrm{~A}$ ). The latter is either oxidized at the electrode surface or diffuses out to the medium, in appearance effluxing from the sensor. If the free movement of any of these analytes is restricted, then there could be an impact on sensor performance. Using glass beads (50-80$\mu \mathrm{m}$ diameter) to simulate physical attributes of the cell cluster, a positive increase in $\Delta \mathrm{i}$ was observed as the sensor approaches the bead surface (Figure 5B). One might anticipate that the presence of the bead could restrict both glucose and oxygen diffusion toward the sensor. If this were the case, the $\Delta \mathrm{i}$ should become more negative on approach, meaning that the apparent glucose signal near the bead ( $\left.\mathrm{i}_{\text {near }}\right)$ was smaller than away $\left(\mathrm{i}_{\text {far }}\right)$. However, the opposite was observed: the $\Delta \mathrm{i}$ was positive (Figure $5 B)$. This would indicate that the sensor signal was more influenced by distortion of hydrogen peroxide efflux from the sensor than by any interference from glucose or oxygen access. The glass bead may be restricting the hydrogen peroxide diffusion into the bulk while enhancing hydrogen peroxide diffusion toward the electrode surface. This interpretation is supported by the effect of adding catalase to the medium ( 2000 units $/ \mathrm{mL}$ ), encouraging hydrogen peroxide to produce water and oxygen as follows:22,23

$$
2 \mathrm{H}_{2} \mathrm{O}_{2} \stackrel{\text { catalase }}{\longrightarrow} 2 \mathrm{H}_{2} \mathrm{O}+\mathrm{O}_{2}
$$

Catalase has been employed by Wittstock to "sharpen" the image of GOx activity obtained from a scanning electrochemical microscope. ${ }^{24}$ Upon catalase introduction to a bulk medium with $5 \mathrm{mM}$ glucose, the positive current recorded near the bead is diminished

(22) Chance, B.; M aehley, A. Assay of Catalases and Peroxidases. In M ethods in Enzymology, II; Colowick, S., Kaplan, N., Eds.; Academic Press: New York, 1955; p 764

(23) Chance, B.; Oshino, N. Biochem. J. 1973, 131, 564-567.

(24) Wittstock, G.; Schuhmann, W. Anal. Chem. 1997, 69, 5059-5066.
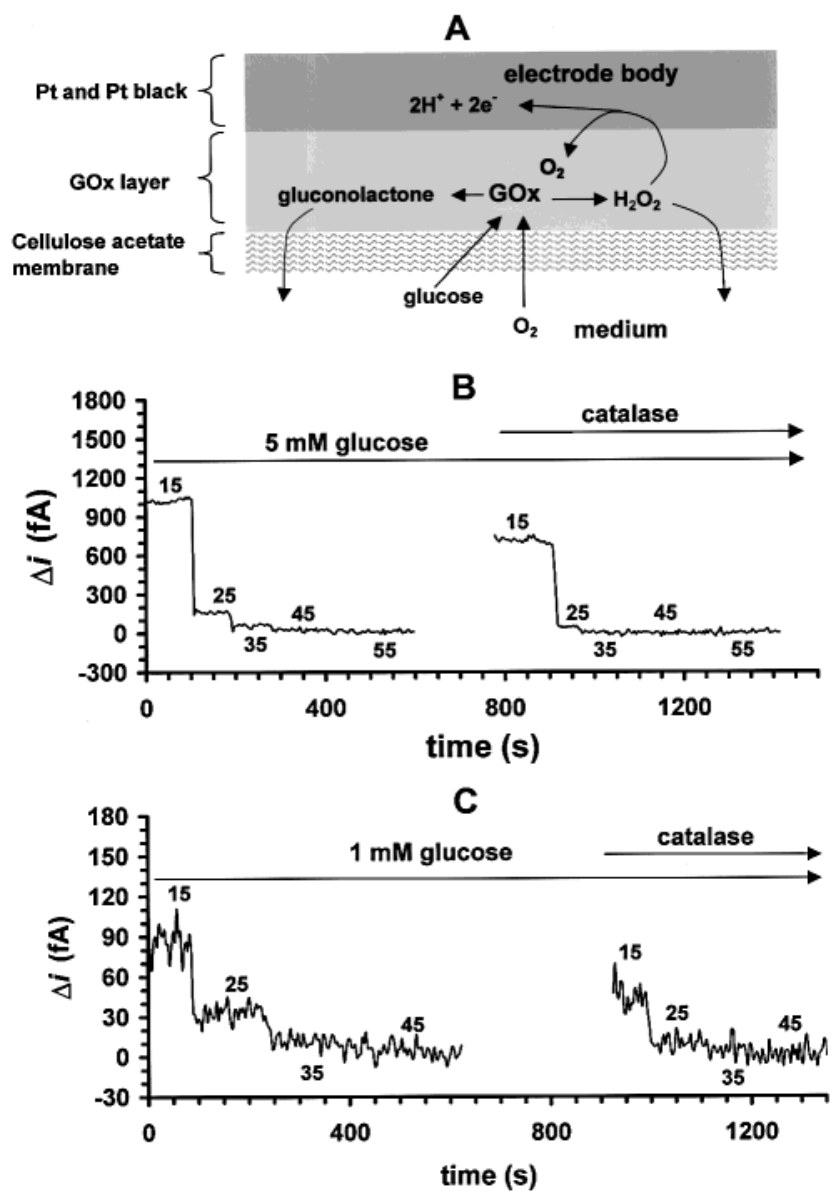

Figure 5. Complication from boundary conditions in the application of the glucose sensor to flux measurement. (A) Schematic to show the chemical movements through the sensor during glucose detection. $(\mathrm{B}, \mathrm{C})$ Artifacts associated with the glucose measurements in proximity to a glass bead. The data shown here are representative of nine measurements at $5 \mathrm{mM}$ glucose $(B)$ and five measurements at $1 \mathrm{mM}$ glucose $(C)$. The final catalase concentration applied in the medium was 2000 units $/ \mathrm{mL}$. The numbers near the traces indicate the mean location of the sensor relative to the glass bead in the self-referencing mode with a $20-\mu \mathrm{m}$ excursion. The artifact is evident as a positive $\Delta i$ on approach to the bead, with an amplitude directly related to the concentration of glucose in the medium. The addition of catalase ameliorates the artifact.

by $29 \pm 5 \%(n=9 ;$ Figure $5 B)$ during self-referencing. The size of the boundary artifact is directly related to glucose concentration, as will be the magnitude of the $\mathrm{H}_{2} \mathrm{O}_{2}$ production (Figure $5 \mathrm{~B}$ and $C)$. The use of catalase presents a method for reducing the boundary artifact, and although the use of the enzyme does not eliminate the artifact, its presence allows glucose measurements at closer proximity to a biological target. As shown in Figure 3B, individual sensors can have a different current output, reflecting the consumption of glucose and $\mathrm{H}_{2} \mathrm{O}_{2}$ production. Therefore, the depth of this artifactual boundary layer will be electrode specific, requiring assessment against a glass sphere prior to use. It is unlikely that the presence of catalase in the medium will affect the biology of the HIT cell clusters as this enzyme exists in the normal environment of cells and is frequently added to cell cultures at similar concentrations. ${ }^{25,26}$

Application of the Self-Referencing Glucose Sensor to the Flux Measurement around a B iological Specimen: Glucose

(25) Ogata, M. Hum. Genet. 1991, 86, 331-340. 

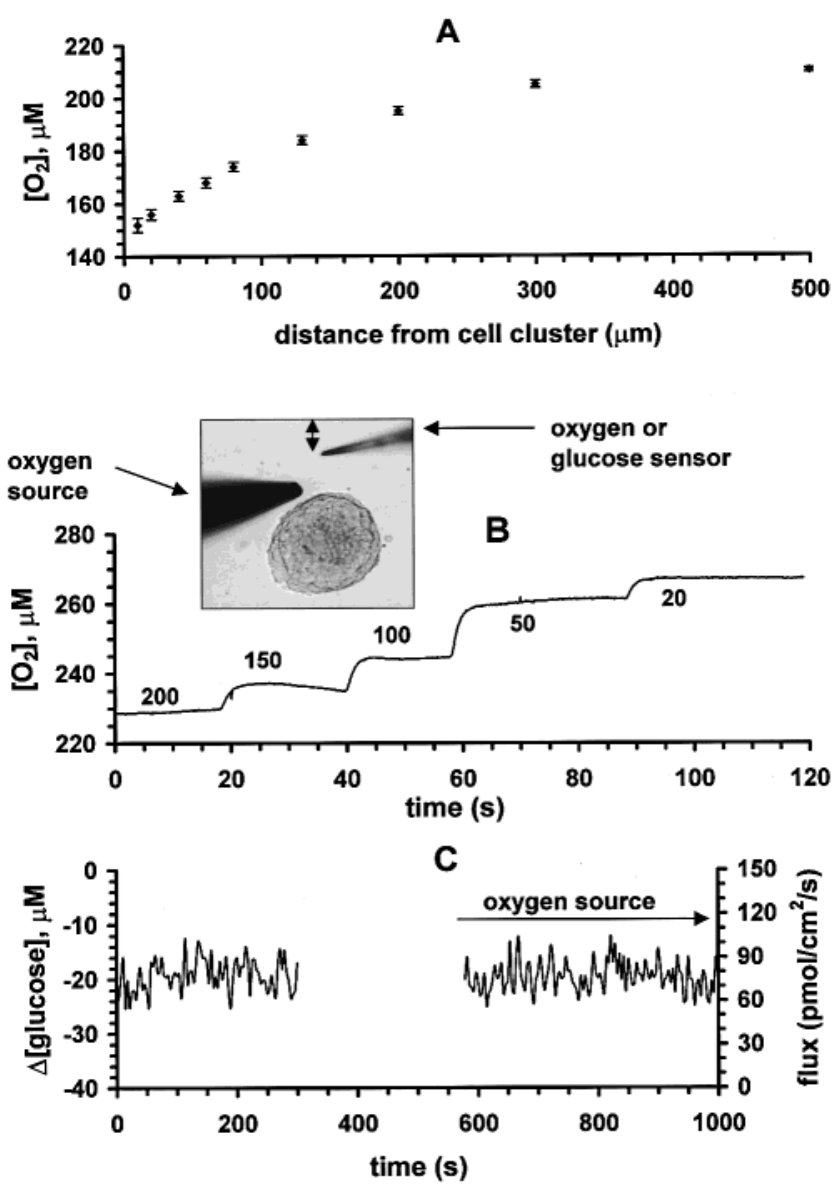

Figure 6. Application of the self-referencing glucose sensor to pancreatic HIT cell clusters. (A) Biological oxygen gradient around a cluster measured in the stationary mode. The data are given as means $\pm \operatorname{SD}(n=4)$. (B) Altered oxygen gradient around a cell cluster in the presence of an oxygen source. The measurement was made in the stationary mode. Inset shows the sensor position relative to the cluster and the oxygen source. The arrow with two end points shows the direction of sensor translation. The numbers near the oxygen trace are the relative distances in micrometers to the cluster. Note that oxygen levels were reversed in the presence of the oxygen source. (C) Glucose uptake measurements and independence from the oxygen gradient. The glucose sensor was self-referenced at 0.2 $\mathrm{Hz}$ between 60 and $80 \mu \mathrm{m}(\Delta r$ of $20 \mu \mathrm{m})$ from the cluster surface. The glucose sensor, oxygen source, and cluster are located as shown in (B). A clear glucose signal is detectable, indicating uptake by the cluster, and shows no change when the respiratory oxygen gradient is reversed. All measurements were made with $5 \mathrm{mM}$ glucose in the medium. The trace shown in (C) is representative of data from five different clusters.

Consumption by Pancreatic $\beta$-Cells. In Figure $3 \mathrm{C}$ we demonstrated that in $5 \mathrm{mM}$ glucose the glucose sensor output was independent of oxygen concentration above a bulk concentration of $70 \mu \mathrm{M}$. However, since the mechanism of glucose detection involves oxygen (see eqS 1 and 2) and cells consume oxygen, knowledge of the oxygen gradient around cells should be a prerequisite for the glucose measurement. To achieve this, an oxygen sensor was used to map the oxygen gradient in proximity to cell clusters with $5 \mathrm{mM}$ glucose in the bulk medium (Figure $6 \mathrm{~A})$. A clear oxygen gradient was evident as expected for

(26) Kaal, E. C. A.; Veldman, H.; Sodaar, P.; Joosten, E. A. J.; Bar, P. R. D. J. Neurosci. Res. 1998, 54, 778-786. aerobically respiring cells. The gradient extended out into the bulk by $300 \mu \mathrm{m}$, but even within $10 \mu \mathrm{m}$ of the cluster surface, the dissolved oxygen concentration did not fall below $140 \mu \mathrm{M}$. Subsequent glucose measurements should, therefore, be independent of these changes in the oxygen concentration.

The glucose measurements were performed by self-referencing between 60 and $80 \mu \mathrm{m}$ ( $\Delta \mathrm{r}$ equal to $20 \mu \mathrm{m}$ ) from the edge of the cluster to avoid the $\mathrm{H}_{2} \mathrm{O}_{2}$ boundary artifact (Figure $6 \mathrm{C}$ ). As shown in Figure $6 \mathrm{~A}$, the oxygen level at $60 \mu \mathrm{m}$ from the clusters was $169 \pm 2 \mu \mathrm{M}$ (means $\pm \mathrm{SD}, \mathrm{n}=4$ ). To further ensure that the apparent glucose signal is due to the glucose gradient and not due to the oxygen gradient, we provided oxygen diffusing from an $\sim 10-\mu \mathrm{m}$ pipet near the cell cluster to reverse the biological oxygen gradient (Figure 6B). Glucose uptake by the $\beta$-cell cluster was clearly evident and did not appear to be affected by the presence of additional oxygen from the source (Figure $6 \mathrm{C}$ ). This observation is reminiscent of those by Wilson and Kennedy and their colleagues in that glucose signal is not affected by oxygen fluctuation as long as the oxygen level remained higher than a certain threshold level. This will vary depending on sensor linearity. ${ }^{6,17}$ The glucose sensor used for the current studies responds linearly up to $10 \mathrm{mM}$ glucose. The $5 \mathrm{mM}$ background glucose lies within this linearity region. In addition, in situ postexperimental calibrations were performed as described in the Experimental Section to document any changes in sensitivity. Accepted were data from drift conditions of less than $\pm 5 \%$ in 60 $\min$.

The flux calculation using eqs 3 and 4 is based on the assumption that the gradient between two measured points is relatively linear and that the sensor is sufficiently far from the surface to allow free diffusion to and from the surface. Addressing the latter point, the ratio of distance to the biological surface versus the modulation distance is 3.5 , satisfying the criteria proposed for SECM . ${ }^{11}$ Demonstrating the relative linearity of the gradient presents problems with potentially nonuniform biological sources. However, studies on ion fluxes from mouse zygotes, similar in radius to the $\beta$-cell cluster, demonstrate a diffusion-based decline defined as $1 / r^{2}$, where $r$ is measured from the center of cell. ${ }^{27}$ Concentration will decline as $1 / r$. In the case of a cluster, measurements are being made at a mean distance of 105-220 $\mu \mathrm{m}$ from the center depending on the cluster diameter. At these distances, the error in assuming linearity should be negligible.

Since a glucose sensor is potentially sensitive to any oxidizable species, such as hydrogen peroxide, ascorbate, or urate, ,,28 biological production of such species could produce signals open to misinterpretation. To test for potential oxidative interferences, an electrode, fabricated in a manner identical to the glucose sensor but without glucose oxidase, was operated in the self-referencing mode. No signals were detected from four cell clusters under the conditions used to obtain the glucose consumption measurements with the glucose sensor (not shown). From these results we conclude that self-referencing a glucose sensor $60 \mu \mathrm{m}$ from a cell cluster of $70-300-\mu \mathrm{m}$ diameter in a medium with $5 \mathrm{mM}$ glucose provides a measure of glucose uptake.

Given that the flux information gathered is a true measure of glucose uptake, and assuming that uptake is evenly distributed

(27) Trimarchi, J. R.; Lin, L.; Smith, P. J. S.; Keefe, D. L. Biol. Prod. 2000, 63, 851-857.

(28) Jung, S.-K.; Wilson, G. S. Anal. Chem. 1996, 68, 591-596. 
over the exposed surface of the cluster, which is very likely the case as the cluster was grown from one cancer cell, it is possible to calculate approximate glucose consumption for the entire cluster and compare this value to those available in the literature. Pancreatic $\beta$-cells in culture attempt to form islets, rounding up as spheroids, allowing an estimate of cluster volume by assuming a shape equivalent to an oblate spheroid, where the lateral radius is symmetrical $\left(R_{1}\right)$ and larger than the vertical $\left(R_{2}\right) . R_{2}$ was estimated by using the motion controller to bring a probe touching the cluster surface to the cover slip. $R_{2}$ was found to be $\sim 0.8 R_{1}$. In the following calculations, the portion of the spheroid resting on the cover slip is not compensated for. The cluster volume (V) is calculated as

$$
V=4 / 3\left[\pi\left(R_{1}^{2} R_{2}\right)\right]
$$

However, as flux values are measured during self-referencing between $\mathrm{x}$ and $(\mathrm{x}+\Delta \mathrm{r}) \mu \mathrm{m}$, where $\mathrm{x}$ is the distance from the cluster surface, the apparent surface area (SA) will be

$$
\mathrm{SA}=4 \pi\left[\left(2 \mathrm{R}_{1}+\mathrm{R}_{2}\right) / 3+\mathrm{x}+\Delta \mathrm{r} / 2\right]^{2}
$$

Flux multiplied by the surface area at the collection point gives a measure of oxygen consumption which can now be related to the volume of the cluster. By following this calculation, measurements acquired from a total of nine cell clusters of $71-140 \mu \mathrm{m}$ in diameter, render basal glucose consumption per unit volume of $61.7 \pm 9.5 \mathrm{fmol} \cdot \mathrm{nL}^{-1} \cdot \mathrm{s}^{-1}$. This value is in agreement with data available from Freyer and Sutherland, who studied the rate of glucose consumption by EMT6/Ro mouse tumor cell clusters using an absorption measurement at $340 \mathrm{~nm}$ with an enzyme reagent kit, observing a consumption of $\sim 48 \mathrm{fmol} \cdot \mathrm{nL}^{-1} \cdot \mathrm{s}^{-1}$ at 5.5 $\mathrm{mM}$ glucose. ${ }^{29}$

Application of the Self-R eferencing Glucose Sensor to the Flux Measurement around a Biological Specimen: Pharmacological Manipulation of Glucose Consumption. In the presence of the oxygen source, glucose consumption was modulated with a protonophore FCCP (Figure 7A), a compound known to increase oxygen consumption by depolarizing the mitochondrial membrane potential and increasing the rate of electron transfer and the consumption of NADH from the Krebs cycle and concomitant glucose consumption. ${ }^{30}$ Before FCCP introduction, a glucose influx of $\sim 40 \mathrm{pmol} \cdot \mathrm{cm}^{-2} \cdot \mathrm{s}^{-1}$ was obtained at $70 \mu \mathrm{m}$ from the cluster. After FCCP introduction, the glucose flux transiently increased and then decreased to below basal. The initial effect increased the level of glucose flux by $93 \pm 16 \%$ subsequently falling after $3 \mathrm{~min}$ to $56 \pm 6 \%$ of the basal condition $(n=5)$. The decrease in glucose consumption following the initial rise could be due to the deleterious effects of FCCP. ${ }^{31} \mathrm{~N}$ ote that the time lag in Figure 7A includes the diffusion time for the FCCP to reach the cells after its application as $0.5 \mu \mathrm{L}$ of $10 \mu \mathrm{M}$ stock solution $\sim 5$ $\mathrm{mm}$ from the cluster. Similar respiratory metabolic responses are observed with the oxygen sensor in the absence of the oxygen source ( $n=4$; not shown). A control experiment confirmed that

(29) Freyer, J. P.; Sutherland, R. M. J. Cell Physiol. 1985, 124, 516-524.

(30) Gunter, T. E.; Pfeiffer, D. R. Am. J. Physiol. 1990, 258, C755-C786.

(31) Dispersyn, G.; N uydens, R.; Connors, R.; Borgers, M .; Geerts, H. Biochim. Biophys. Acta 1999, 1428, 357-371.
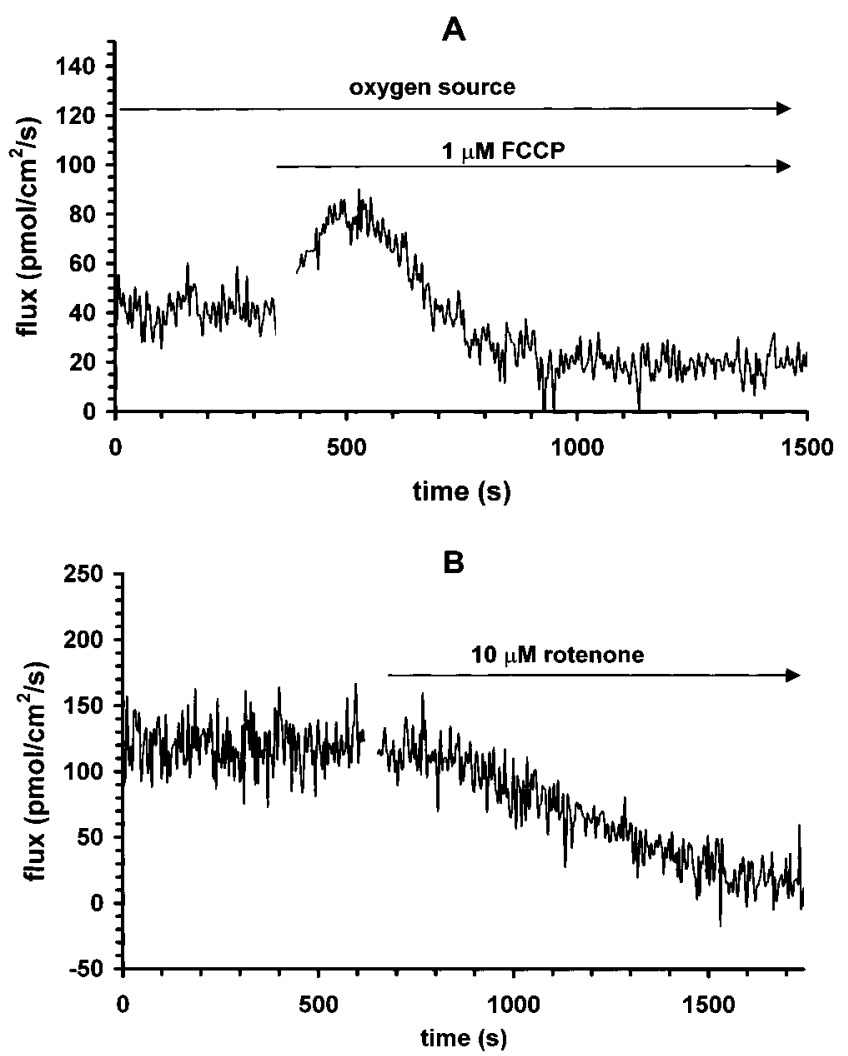

Figure 7. (A) Influence of $1 \mu \mathrm{M}$ FCCP on glucose flux around a cell cluster. The glucose sensor was self-referenced at $0.2 \mathrm{~Hz}$ between 60 and $80 \mu \mathrm{m}(\Delta r$ of $20 \mu \mathrm{m})$ from the cluster surface. The glucose sensor, oxygen source, and cell cluster are located as illustrated in Figure 6B. The aliquot of FCCP was dropped on the meniscus of the medium to minimize disturbance of the existing glucose gradient. All measurements were performed with $5 \mathrm{mM}$ glucose in the bath. The trace shown is representative of five different cluster measurements. FCCP acts to initially increase glucose uptake, subsequently falling to below basal after $\sim 3 \mathrm{~min}$. (B) Influence of 10 $\mu \mathrm{M}$ rotenone on glucose flux around the cell cluster. The glucose sensor was self-referenced between 60 and $80 \mu \mathrm{m}(\Delta r$ of $20 \mu \mathrm{m})$ at $0.2 \mathrm{~Hz}$. The rotenone aliquot was dropped on the meniscus of the medium to minimize disturbance of the existing glucose gradient. The measurement was performed with $5 \mathrm{mM}$ glucose in the bath. The trace shown is representative of four different cluster measurements. Rotenone acts to reduce the level of glucose consumption by $\sim 75 \%$ after $10 \mathrm{~min}$.

the glucose sensor performance is not affected by the presence of FCCP (not shown).

Glucose metabolism was further studied with rotenone, an inhibitor of NADH-dependent respiration in mitochondria. ${ }^{32}$ Rotenone induced a $74 \pm 5 \%$ decrease in the glucose flux $(n=4$; Figure 7B). With regard to the lag period between application and response, the same interpretation applies as discussed above for FCCP. During these experiments, an abundant supply of oxygen was available via the artificial source positioned near the cluster. A control experiment confirmed that the glucose sensor performance was not affected by the presence of rotenone (not shown).

\section{CONCLUSIONS}

In conclusion, the results of this study have demonstrated that a sensor incorporating an immobilized enzyme, in this case

(32) Soloway, S. B. Environ. Health Perspect. 1976, 14, 109-117. 
glucose oxidase, can be used in a self-referencing mode. As with previous applications of self-referencing sensors for both ion detection ${ }^{10,18}$ and gases, such as oxygen and nitric oxide, ${ }^{8,9}$ the self-referencing approach greatly enhances the detection sensitivity by virtue of reducing the impact of drift. Our ability to follow the glucose transport process in the pancreatic $\beta$-cell has the added importance of providing an insight into the mechanisms underlying insulin secretion. Central to the control of secretion is the concept of a cellular glucose sensor and its regulatory role. ${ }^{15}$ Further, downregulation of the glucose transporter in these cells correlates with decreased glucose uptake and the loss of insulin secretion in type II diabetes. ${ }^{33-35}$ The methodology reported here offers real-time access to changes in the glucose microenvironment surrounding these cells although currently confined to observations on small clusters of $\sim 300$ cells. Recently, however, the same procedure has been shown to provide glucose transport

(33) Unger, R. H. Science 1991, 251, 1200-1205.

(34) Burcelin, R.; Dolci, W.; Thorens, B. Diabetes 2000, 49, 1643-1648.

(35) Thorens, B.; Guillam, M . T.; Beermann, F.; Burcelin, R.; Jaquet, M . J. Biol. Chem. 2000, 275, 23751-23758. data from single cardiac myocytes (J ung, Smith, and M cDonough, unpublished).

Preliminary results (J ung and Smith, unpublished) indicate that diversification to other enzyme-based reactions, such as lactate and glutamate oxidases, will be straightforward.

\section{ACKNOWLEDGMENT}

We thank Jane M cLaughlin, Kasia Hammar, and Robert Lewis for technical assistance. We also thank Yaney Gordon of Boston University, M edical School, for guidance with the cell culture. The work was supported by NIH Grant P41 RR01395 (NCRR to P.J.S.S.) and the Lalor Foundation (J.R.T.). The BioCurrents Research Center is a national resource of the NIH providing access to specialized techniques for the study of transport physiology.

Received for review February 28, 2001. Accepted May 30, 2001.

AC010247U 